(January 1996)

\title{
Axial Anomaly and the Nucleon Spin
}

\author{
Jin Hu Lin 7 and C. S. Kim! \\ Department of Physics, Yonsei University, \\ Seoul 120-749, Republic of Korea
}

\begin{abstract}
In this letter we have taken a particular Lagrangian, which was introduced to resolve $U(1)$ problem, as an effective QCD Lagrangian, and have derived a formula of the quark content of the nucleon spin. The difference between quark content of the proton $\left(\Delta \Sigma_{p}\right)$ and that of the neutron $\left(\Delta \Sigma_{n}\right)$ is evaluated by this formula. Neglecting the higher-order isospin corrections, this formula can reduce to Efremov's results in the large $N_{c}$ limit.
\end{abstract}

*Permanent address : Dept. of Physics, Yanbian Univ., Jilin Prov., China

${ }^{\dagger}$ E-mail address : kim@cskim.yonsei.ac.kr, cskim@kekvax.kek.jp 


\section{Introduction}

The EMC data [1] taken in conjunction with the Bjorken sum rule [2] imply that the

total spin carried by the quarks in the polarized proton amounts to only about $\frac{1}{8}$ of the spin of the proton. One possible way to explain these surprising results is to take into account a quantum effect known as the "axial anomaly" of QCD. The anomaly makes it possible for spin carried by gluons to mix with spin carried by quarks, thus modifying the structure of the quark sea. Effectively, if the gluon polarization is big, i.e. if the amount of spin carried by the gluon is large and positive, the fraction of the nucleon spin carried by quarks will appear to be smaller than it really is. Thus the existence of a large anomaly effect would explain the smallness of the apparent quark contribution to the proton spin. Six years ago, Cheng and Li [3] suggested, looking at the anomaly effect of the $U(1)$ Ward identity,

$$
\partial_{\nu} J_{\nu}^{5}=\sum_{i} 2 m_{i} \bar{q}_{i} i \gamma_{5} q_{i}+\partial_{\nu} \widetilde{K}_{\nu}
$$

where $J_{\nu}^{5}$ is the axial-vector singlet quark current, and $\widetilde{K}_{\nu}$ is the topological current. From Eq. (11), there seems to be a natural seperation of the current matrix element into the quark contribution (the first term), which is hoped to yield the naive quark-model result, and the gluon anomaly contribution (the second). However, the calculated gluon contribution is opposite in sign to what one had expected in the parton model [4]. Actually, in any covariant gauge

$$
\begin{aligned}
\left\langle p^{\prime}\left|J_{\nu}^{5}\right| p\right\rangle & =\bar{u}\left(p^{\prime}\right)\left[\gamma_{\nu} \gamma_{5} G_{1}\left(q^{2}\right)+q_{\nu} \gamma_{5} G_{2}\left(q^{2}\right)\right] u(p) \\
\left\langle p^{\prime}\left|\widetilde{K}_{\nu}\right| p\right\rangle & =\bar{u}\left(p^{\prime}\right)\left[\gamma_{\nu} \gamma_{5} \widetilde{G}_{1}\left(q^{2}\right)+q_{\nu} \gamma_{5} \widetilde{G}_{2}\left(q^{2}\right)\right] u(p)
\end{aligned}
$$

where $q=p-p^{\prime}, \bar{u}$ and $u$ are the polarized proton wave functions, and $G_{i}\left(\widetilde{G}_{i}\right)^{\prime}$ s are form factors. Above expressions can be written in the form, when $p^{\prime}=p$,

$$
\begin{aligned}
\left\langle p^{\prime}\left|J_{\nu}^{5}\right| p\right\rangle & =2 M_{N} S_{\nu} G_{1}(0), \\
\left\langle p^{\prime}\left|\widetilde{K}_{\nu}\right| p\right\rangle & =2 M_{N} S_{\nu} \widetilde{G}_{1}(0),
\end{aligned}
$$


where $M_{N}$ is the nucleon mass, $S_{\nu}$ its spin four-vector. And according to definition of Efremov et. al. [5], the contributions parallel to it are, in terms of quark and gluon distributions

$$
G_{1}(0)=\Delta \Sigma-\Delta \widetilde{g} \quad \text { and } \quad \widetilde{G}_{1}(0)=-\Delta \widetilde{g}, \quad\left(\Delta \widetilde{g}=\frac{\alpha_{s}}{2 \pi} N_{f} \Delta g\right)
$$

Thus, the calculated gluon distribution is large and positive. However, they neglected the large isospin violation and assumed that $\Delta m_{\eta^{\prime}} g_{Q N N} \ll g_{\eta^{\prime} N N}=6.3$ in the final calculation. We note the following two points. First, the term proportional to $\left(m_{u}-m_{d}\right)$ is neglected in Ref. [5], due to it leads to higher-order isospin corrections. But, according to Ref. [3], the size of the term contributing to $\Delta \Sigma_{p}$ is about 0.38. Neglecting thus large contribution, the obtained results are not satisfactory. Second, Kochelev [0] has shown that the the contribution of the $\pi^{0}$-ghost mixing to the charge symmetry breaking (CSB) may be significant, and the value of CSB is determined by the mass difference of $d$ - and $u$-quarks and the ghost-nucleon coupling constant $g_{Q N N}$. If we determin the value of CSB in the different methods, then the ghost-nucleon coupling constant $g_{Q N N}$ can still be obtained. Therefore, the assumption of $\Delta m_{\eta^{\prime}} g_{Q N N}<<g_{\eta^{\prime} N N}$ is not always necessary.

In this letter we take aim at solving above two questions. The paper organized as follows. In Section 2, we will start from a particular Lagrangian, and derive a formula of the quark content of nucleon spin. In Section 3, the difference between $\Delta \Sigma_{p}$ and $\Delta \Sigma_{n}$ is evaluated. Neglecting the higher-order corrections, we found it comparable with the value of Ref. [5]. In Section 4, the value of the ghost-nucleon coupling constant is obtained by introducing charge symmey beaking in the pionnucleon coupling. Finally, discussions and conclusions are given in Section 5.

\footnotetext{
${ }^{1}$ Here we would like to remind the readers that the definition of quark and gluon distribution function is renormalization scheme dependent [6]. Based on the gauge-invariant operator product expansion, the first moment of the flavor-singlet polarized distribution function is given by $\Delta \Sigma(=$ $\left.G_{1}(0)\right)$, which is equal to $\Delta \Sigma-\Delta \widetilde{g}$ in this paper, as shown in (4).
} 


\section{The effective Lagrangian and a formula of the quark content in the nucleon spin}

The longstanding $U(1)$ problem (including not only $\eta$ mass problem, but also problem of $\eta \rightarrow \pi^{+} \pi^{-} \pi^{0}$ decay) can be consistently resolved in the follwing effectve Lagrangian, where $U(1)$ anomaly is taken into accunt as $O\left(\frac{1}{N_{c}}\right)$ effect [8] :

$$
\mathcal{L}=\frac{F_{\pi}^{2}}{16} \operatorname{Tr}\left[\partial_{\mu} U \partial^{\mu} U^{\dagger}\right]+\sum_{a}^{0,3,8} c^{a} u^{a}+\frac{1}{2 F_{s}^{2} \widetilde{m}^{2}}\left(\partial^{\mu} \widetilde{K}_{\mu}\right)^{2}-\frac{1}{F_{s}}\left(\partial^{\mu} \widetilde{K}_{\mu}\right) S
$$

with

$$
U=\exp \left[i \frac{2}{F_{\pi}}\left(\lambda^{0} S+\lambda^{a} \pi^{a}\right)\right], \quad u^{a}=\frac{1}{4} \operatorname{Tr}\left[\lambda^{a}\left(U+U^{\dagger}\right)\right]
$$

where $\lambda^{a}$ are the usual Gell-Mann matrices, $F_{\pi}$ is the pion decay constant with

input value of $F_{\pi}=186.4 \mathrm{MeV}$, and $F_{s}=\sqrt{\frac{3}{2}} F_{\pi} \sim O\left(\sqrt{N_{c}}\right)$, and $\pi^{a}(S)$ is the flavor octet (singlet) pseudoscalar field. The explicit $S U_{f}(3)$ breaking is represented by $\left(c^{0}, c^{8}, c^{3}\right)=\left(\frac{1}{4} \sqrt{\frac{3}{2}} F_{\pi}^{2} m_{N_{s}}^{2},-\frac{F_{\pi}^{2}}{2 \sqrt{3}}\left(m_{K}^{2}-m_{\pi}^{2}\right), \frac{1}{4} F_{\pi}^{2} \delta m^{2}\right)$ with $m_{N_{S}}^{2}=\frac{1}{3}\left(2 m_{K}^{2}+m_{\pi}^{2}\right)$ and $\delta m^{2}=m_{K^{+}}^{2}-m_{K^{0}}^{2}-m_{\pi^{+}}^{2}+m_{\pi^{0}}^{2}$. The last two terms in Eq. (5) contain an axial vector ghost field $\widetilde{K}_{\mu}$, which add a mass $\widetilde{m}^{2}=m_{\eta^{\prime}}^{2}+m_{\eta}^{2}-2 m_{K}^{2} \sim O\left(\frac{1}{N_{c}}\right)$ to the $U(1)$ Nambu-Goldston boson $S$. Here $\widetilde{K}_{\mu}$ in Eq. (可) should be identified with a non-perturbatie realization of the topological current in QCD

$$
\widetilde{K}_{\mu}=N_{f} \frac{\alpha_{s}}{2 \pi} \epsilon_{\mu \nu \rho \sigma} A_{\rho}^{a}\left(\partial_{\sigma} A_{\rho}^{a}-\frac{1}{3} g f_{a b c} A_{\sigma}^{a} A_{\rho}^{c}\right)
$$

In the effective Lagrangian, the anomaly Eq. (11) takes the form

$$
\partial^{\mu} J_{\mu}^{5}=2 \sum_{a}^{0,3,8} c^{a} v^{a}+\partial^{\mu} \widetilde{K}_{\mu}
$$

with

$$
v^{a}=\frac{1}{4} i \operatorname{Tr}\left[\lambda^{a}\left(U-U^{\dagger}\right)\right]
$$

By substituting Eq. (2) into Eq. (8) one gets

$$
2 M_{N} G_{1}\left(q^{2}\right)+q^{2} G_{2}\left(q^{2}\right)=\lambda+2 M_{N} \widetilde{G}_{1}\left(q^{2}\right)+q^{2} \widetilde{G}_{2}\left(q^{2}\right)
$$


where

$$
\begin{aligned}
& \lambda=\frac{\left\langle p^{\prime}\left|\sum_{a}^{0,3,8} 2 c^{a} v^{a}\right| p\right\rangle}{\bar{u}\left(p^{\prime}\right) i \gamma_{5} u(p)} \\
& =\frac{4}{F_{\pi}}\left(c_{0}, c_{8}, c_{3}\right)\left(\begin{array}{ccc}
\cos \theta_{3} & -\sin \theta_{3} & \theta_{2} \\
\sin \theta_{3} & \cos \theta_{3} & -\theta_{1} \\
\theta_{1} \sin \theta_{3}-\theta_{2} \cos \theta_{3} & \theta_{1} \cos \theta_{3}+\theta_{2} \sin \theta_{3} & 1
\end{array}\right)\left(\begin{array}{c}
\frac{g_{\eta^{\prime} N N}}{m_{\eta^{\prime}}^{2}} \\
\frac{g_{\eta N N}}{m_{\eta}^{2}} \\
\pm \frac{g_{\pi^{0} N N}}{m_{\pi^{0}}^{2}}
\end{array}\right) \\
& =\frac{4}{F_{\pi}}\left\{g_{\eta^{\prime} N N} m_{\eta^{\prime}}^{2}\left[c_{0} \cos \theta_{3}+c_{8} \sin \theta_{3}+c_{3}\left(\theta_{1} \sin \theta_{3}-\theta_{2} \cos \theta_{3}\right)\right]\right. \\
& +g_{\eta N N} m_{\eta}^{2}\left[-c_{0} \sin \theta_{3}+c_{8} \cos \theta_{3}+c_{3}\left(\theta_{1} \cos \theta_{3}+\theta_{2} \sin \theta_{3}\right)\right] \\
& \left. \pm g_{\pi^{0} N N} m_{\pi^{0}}^{2}\left(c_{0} \theta_{2}-c_{8} \theta_{1}+c_{3}\right)\right\} \text {, }
\end{aligned}
$$

where $\theta_{1}, \theta_{2}, \theta_{3}$ are the mixing angles between $\pi^{0}$ and $\eta, \pi^{0}$ and $\eta^{\prime}, \eta$ and $\eta^{\prime}$, respectively. The parameter $g$ 's are the coupling constants. In the limit $q^{2} \rightarrow 0$, and $q^{2} G_{2}\left(q^{2}\right) \rightarrow 0$, then $\lim _{q^{2} \rightarrow 0} q^{2} \widetilde{G}_{2}\left(q^{2}\right)=G=F_{s}\left(\widetilde{m}^{2} I-\widetilde{m} g_{Q N N}\right)$ is the residue of

the ghost pole contributin [5], where $I=\frac{g_{\eta^{\prime} N N}}{m_{\eta^{\prime}}^{2}} \cos \theta_{3} \pm \frac{g_{\pi^{0} N N}}{m_{\pi^{0}}^{2}} \theta_{2}-\frac{g_{\eta N N}}{m_{\eta}^{2}} \sin \theta_{3}$. (Due to $\pi^{0}-\eta-\eta^{\prime}$ mixing, instead of one $\eta^{\prime}$ pole we have to put the singlet combination of $\eta^{\prime}, \eta, \pi^{0}$ poles. Thus, we substitute $\widetilde{m}$ for $\Delta m_{\eta^{\prime}}$ in the expression of $G$. In our numerical calculation we take $\widetilde{m}^{2}=\Delta m_{\eta^{\prime}}^{2} \approx 0.726 \mathrm{GeV}^{2}$.) In the expressions of $\lambda$ and $I$, the plus or minus sign is for proton or neutron, respectively. One can re-express Eq. (10) at $q^{2}=0$ as

$$
G_{1}(0)=\widetilde{G}_{1}(0)+\frac{1}{2 M_{N}}(\lambda+G)
$$

Let $\Delta \widetilde{g}=-\widetilde{G}_{1}(0)$ as in Ref. [5], then one find

$$
\Delta \Sigma=\frac{1}{2 M_{N}}(\lambda+G)=\frac{1}{2 M_{N}}\left[\lambda+F_{s}\left(\widetilde{m}^{2} I-\widetilde{m} g_{Q N N}\right)\right]
$$

This is a formula of the quark content in the nucleon spin. Neglecting the higherorder isospin corrections, Eq. (13) can reduce to Eq. $\left(24^{\prime}\right)$ in Ref. [5]. (For more details, see Appendix.) 


\section{Difference between $\Delta \Sigma_{p}$ and $\Delta \Sigma_{n}$}

To calculate the difference between quark content of the proton $\left(\Delta \Sigma_{p}\right)$ and that of the neutron $\left(\Delta \Sigma_{n}\right)$, we only take amount into terms that carry the plus and minus signs in Eq. (13),

$$
\begin{aligned}
\Delta \Sigma_{p}-\Delta \Sigma_{n}= & \frac{1}{2 M_{N}}\left\{2 F_{s} \widetilde{m}^{2} \frac{g_{\pi^{0} N N}}{m_{\pi^{0}}^{2}} \theta_{2}+\frac{2 F_{\pi} g_{\pi 0}{ }^{0} N}{m_{\pi^{0}}^{2}}\left[\sqrt{\frac{3}{2}} \frac{\left(2 m_{K}^{2}+m_{\pi}^{2}\right)}{3} \theta_{2}\right.\right. \\
& \left.\left.+\frac{2}{\sqrt{3}}\left(m_{K}^{2}-m_{\pi}^{2}\right) \theta_{1}+\delta m^{2}\right]\right\}
\end{aligned}
$$

Due to $\theta_{2}=-\sqrt{\frac{3}{2}} \frac{\delta m^{2}}{m^{2}}$ and $\theta_{1}=-\theta_{2} \frac{m_{\eta^{\prime}}^{2}+m_{\eta}^{2}-2 m_{\pi}^{2}}{2 \sqrt{2}\left(m_{K}^{2}-m_{\pi}^{2}\right)}$, one can re-express Eq. (14) as

$$
\begin{aligned}
\Delta \Sigma_{p}-\Delta \Sigma_{n}= & \frac{1}{2 M_{N}}\left\{2 F_{s} \widetilde{m}^{2} \frac{g_{\pi^{0} N N}}{m_{\pi^{0}}^{2}} \theta_{2}+\frac{2 F_{\pi} g_{\pi^{0} N N}}{m_{\pi^{0}}^{2}}\left[\sqrt{\frac{3}{2}} \frac{\left(2 m_{K}^{2}+m_{\pi}^{2}\right)}{3}\right.\right. \\
& \left.\left.-\frac{1}{\sqrt{6}}\left(m_{\eta^{\prime}}^{2}+m_{\eta}^{2}-2 m_{\pi}^{2}\right)-\sqrt{\frac{2}{3}}\left(\widetilde{m}^{2}\right)\right] \theta_{2}\right\} \\
= & \frac{1}{2 M_{N}}\left\{\frac{2 F_{\pi} g_{\pi^{0} N N}}{\sqrt{6} m_{\pi^{0}}^{2}} 3 m_{\pi}^{2}\right\} \theta_{2} \\
\approx & \frac{3 F_{\pi} g_{\pi^{0} N N}}{\sqrt{6} M_{N}} \theta_{2}=2 \sqrt{\frac{3}{2}} g_{A}^{3} \theta_{2},
\end{aligned}
$$

where we have made use of Goldberger-Treiman relation, i.e. $g_{A}^{3}=\frac{F_{\pi} g_{\pi_{0} N N}}{2 M_{N}}$. It is clear that Eq. (14) reduces to the result of Ref. [5], once neglecting higher-order isospin corrections.

\section{Charge symmetry breaking in the pion-nucleon coupling constant and the value of the ghost- nucleon coupling constant}

Usually one neglects charge symmetry breaking (CSB), since most of popular mechanisms provides a very small contribution. However, the analysis without this supposition [9] leads to a large magnitude of CSB. The Nijmegen group has recently completed the phase shift analysis of all $N N$ scattering data below $E_{\text {lab }}=350$ $\mathrm{MeV}$. This is a continuation of the Nijmegen analysis between $0 \sim 30 \mathrm{MeV}$. Both 
in the $p p$ and $n p$ analysis, a low value for the $\pi N N$ coupling constant was found, indicating a large charge symmetry breaking. They found the following results [10].

$$
\begin{aligned}
G_{p p \pi^{0}} & =g_{0}, & \frac{G_{p n \pi^{+}}}{\sqrt{2}}=g_{0}+\frac{3}{2} \Delta g, \\
-G_{n n \pi^{0}} & =g_{0}+2 \Delta g, & \frac{G_{n p \pi^{-}}}{\sqrt{2}}=g_{0}+\frac{3}{2} \Delta g,
\end{aligned}
$$

where $g_{0}^{2}=\left(\frac{2 m_{p}}{m_{c}}\right)^{2} \times f_{0}^{2}=13.48$ is $p p \pi^{0}$ coupling constant, and $\Delta g^{2}=g_{c}^{2}-g_{0}^{2}=$ $\left(G_{p n \pi^{+}} G_{n p \pi^{-}}\right) / 2-g_{0}^{2}=\left(\frac{m_{p}+m_{n}}{m_{c}}\right)^{2} f_{c}^{2}-13.48=0.06$, with $g_{c}^{2}$ the charged coupling constant.

After solving Eq. (16) we find

$$
\begin{aligned}
& g_{p p \pi^{0}}=g_{0}^{2}=13.48, \\
& g_{n n \pi^{0}}=G_{n n \pi^{0}}^{2}=13.61 .
\end{aligned}
$$

On the other hand, the contribution of the mixing of the $\pi^{0}$-meson and the ghost pole of the $U(1)$ problem to the pion-nucleon coupling constant is obtaind by Kochelev [7]. It is shown that the value of CSB in these constants is defined by the mass difference of $d$-and $u$-quark and the value of the ghost-nuceon coupling constant. According to Ref. [7],

$$
\frac{g_{n n \pi^{0}}^{2}-g_{p p \pi^{0}}^{2}}{2 g_{\pi^{0} N N}^{2}}=\frac{g_{Q N N} m_{\pi}\left(m_{d}-m_{u}\right)}{g_{\pi^{0} N N} \sqrt{m_{d} m_{u}}},
$$

or

$$
g_{Q N N}=\frac{\left(g_{n n \pi^{0}}^{2}-g_{p p \pi^{0}}^{2}\right) \sqrt{m_{d} m_{u}}}{2 g_{\pi^{0} N N} m_{\pi}\left(m_{d}-m_{u}\right)} .
$$

Using Eq. (17) and $\frac{m_{d}-m_{u}}{m_{d}+m_{u}}=0.27 \pm 0.03$ [11], we find the value of the ghost-nucleon coupling constant:

$$
g_{Q N N}=3.49 \times 10^{-3} \mathrm{MeV}^{-1}
$$

\section{Discussions and conclusions}

In this letter we have taken a particular Lagrangian as an effective QCD Lagrangian and have derived a formula of the quark content of the nucleon spin. According to this formula, we have obtained the difference between $\Delta \Sigma_{p}$ and $\Delta \Sigma_{n}$. 
Now we evaluate the numerical value of $\Delta \Sigma$ and $\Delta \widetilde{g}$. The physical masses of mesons are $m_{\eta^{\prime}}=958 \mathrm{MeV}, m_{\eta}=549 \mathrm{MeV}, m_{\pi^{0}}=135 \mathrm{MeV}$, and the mixing angles determined by Ref. [12] are

$$
\theta_{1}=-1.7 \times 10^{-2}, \quad \theta_{2}=0.9 \times 10^{-2}, \quad \theta_{3}=-0.31 .
$$

The $\pi$-decay constant is $F_{\pi}=186.4 \mathrm{MeV}$, and the $\eta^{\prime}$ decay constant is $f_{\eta^{\prime}}=132$ $\mathrm{MeV}$. Using these values, together with the values $g_{\eta^{\prime} N N}=6.3, g_{\eta N N}=6.1, g_{\pi^{0} N N}=$ 13.55, one obtains, from Eq. (13) and Eq. (20)

$$
\Delta \Sigma= \begin{cases}0.66 & \text { for proton } \\ 0.50 & \text { for neutron }\end{cases}
$$

and

$$
\Delta \Sigma_{p}-\Delta \Sigma_{n}=0.16
$$

Frois and Karliner [13] found the fact that in the lowest order of perturbation theory there is an agreement between the neutron and proton experiments, but when higher-order QCD corrections are taken into account, all of the experimental results converge to the value $G_{1}(0) \approx 0.30 \pm 0.11$. Using this result one obtains, from Eq. (12) and Eq. (20)

$$
\Delta \widetilde{g}= \begin{cases}0.36 & \text { for proton } \\ 0.20 & \text { for neutron }\end{cases}
$$

Several comments are now in order. First, neglecting the higher-order corrections, from Eq. (15) one can obtain $\Delta \Sigma_{p}-\Delta \Sigma_{n}=0.03$. This result is much smaller than Eq. (23). It implies that higher-order corrections are non-negligible. Second, another new result of this letter is a detailed calculation of the ghost-necleon coupling constant. The large value $\Delta m_{\eta^{\prime}} g_{Q N N} \approx 2.97$ makes it unreasonable to assume that $\Delta m_{\eta^{\prime}} g_{Q N N} \ll g_{\eta^{\prime} N N} \approx 6.3$, as in Ref. [5]. When ghost pole exchange is taken into account in the OBEP (One Boson Exchange Potentiol) analysis of $N N$ scattering, $g_{\eta^{\prime} N N}$ approaches to $\sqrt{g_{\eta^{\prime} N N}^{2}-m_{\eta^{\prime}}^{2} g_{Q N N}^{2}}$. Due to large value of $m_{\eta^{\prime}}^{2} g_{Q N N}^{2}$, it implies the geat suppression of $g_{\eta^{\prime} N N}$. This result just agrees with the fact that the 
Skyrme model predicts $g_{\eta^{\prime} N N} \approx 0$ in the large $N_{c}$ limit. On the other hand, it is enough to prove that Eq. (13) can reduce to Eq. $\left(24^{\prime}\right)$ in Ref. [5], neglecting the higher-order isospin corrections. It implies that Eq. (13) possesses more general meaning, comparing with Eq. (24') in Ref. [5].

To summarize, in this letter we have taken a particular Lagrangian, which was introduced to resolve $U(1)$ problem, as an effective QCD Lagrangian and have derived a formula of the quark content of the nucleon spin. The difference between quark content of the proton $\left(\Delta \Sigma_{p}\right)$ and that of the neutron $\left(\Delta \Sigma_{n}\right)$ is evaluated by this formula. Neglecting higher-order isospin corrections, this formula can reduce to Efremov's results in the large $N_{c}$ limit.

\section{Acknowledgements}

The work was supported in part by the Korean Science and Engineering Foundation, Project No. 951-0207-008-2, in part by Non-Directed-Research-Fund, Korea Research Foundation 1993, in part by the CTP, Seoul National University, in part

by Yonsei University Faculty Research Grant 1995, and in part by the Basic Science Research Institute Program, Ministry of Education, 1995, Project No. BSRI-952425 . 


\section{References}

[1] J. Ashman el al., Phys. Lett. B206 (1988) 364.

[2] J.D. Bjorken, Phys. Rev. 148 (1966) 1467, Phys. Rev. D1 (1971) 1376.

[3] T.P. Cheng and Ling-Feng Li, Phys. Rev. Lett. 62 (1989) 1441.

[4] G. Altarelli and G.G. Ross, Phys. Lett. B212 (1988) 391; R.D. Carlitz, J.C. Collins, and A. Mueller, Phys. Lett. B214 (1988) 229.

[5] A.V. Efremov, J. Soffer and N.A. Törnqvist, Phys. Rev. D44 (1991) 1369.

[6] A.V. Manohar, Phys. Rev. Lett. 65 (1990) 2511.

[7] N.I. Kochelev, Phys. Lett. B301 (1993) 272.

[8] P. Deviccia and G. Veneziano, Nucl. Phys. B171 (1980) 253; P. Nath and R. Arnowitt, Phys. Rev. D23 (1981) 473; E. Witten, Ann. Phys. (N.Y.) 128 (1980) 363.

[9] R.G.E. Timmermans, Th.A. Rijken and J.J. De Swart, Phys. Rev. Lett. 67 (1991) 1074; V. Bernard and U. G. Meissner, Nucl. Phys. A508 (1990) 361c.

[10] Th.A. Rijken, V.G.J. Stokes, R.A.M. Klomp, J.L. de Kok and J.J. de Swart, Nucl. Phys. A508 (1990) 173c.

[11] B.L. Ioffe, Sov. J. Nucl. Phys. 29 (1979) 827; J. Gasser and H. Leutwyler, Phys. Rev. 87 (1982) 77.

[12] K. Kawarabayashi and N. Ohta, Nucl. Phys. B175 (1980) 477.

[13] B. Frois and M. Karliner, Phys. World. 7 (1994) 44. 


\section{Appendix}

Neglecting higher-order isospin corrections, Eq. (13) can reduce to Eq. (24') of Ref. [5]. The proof is as follows.

First term of Eq. (13) :

$$
\begin{aligned}
\frac{F_{\pi} g_{\eta^{\prime} N N}}{2 M_{N} m_{\eta^{\prime}}^{2}}\left[\cos \theta_{3} \frac{2 m_{K}^{2}+m_{\pi}^{2}}{\sqrt{6}}-\frac{2}{\sqrt{3}}\left(m_{K}^{2}-m_{\pi}^{2}\right) \sin \theta_{3}+\left(m_{K^{+}}^{2}-m_{K^{0}}^{2}-m_{\pi^{+}}^{2}+m_{\pi^{0}}^{2}\right)\right. \\
\left.\quad \times\left(\theta_{1} \sin \theta_{3}-\theta_{2} \cos \theta_{3}\right)\right]+F_{s} \widetilde{m}^{2} \frac{g_{\eta^{\prime} N N}}{m_{\eta^{\prime}}^{2}} \cos \theta_{3} \\
\longrightarrow \frac{F_{\pi} g_{\eta^{\prime} N N}}{2 M_{N} m_{\eta^{\prime}}^{2}}\left[\cos \theta_{3} \frac{2 m_{K}^{2}+m_{\pi}^{2}}{\sqrt{6}}+\sqrt{\frac{3}{2}}\left(m_{\eta^{\prime}}^{2}+m_{\eta}^{2}-2 m_{K}^{2}\right) \cos \theta_{3}-\frac{2}{\sqrt{3}}\left(m_{K}^{2}-m_{\pi}^{2}\right) \sin \theta_{3}\right] \\
\longrightarrow \frac{F_{\pi} g_{\eta^{\prime} N N}}{2 M_{N} m_{\eta^{\prime}}^{2}}\left[\cos \theta_{3} \frac{3 m_{\eta^{\prime}}}{\sqrt{6}}+\frac{\cos \theta_{3}\left(3 m_{\eta^{\prime}}^{2}-4 m_{K}^{2}+m_{\pi}^{2}\right)-2 \sqrt{2}\left(m_{K}^{2}-m_{\pi}^{2}\right) \sin \theta_{3}}{\sqrt{6}}\right] \\
\longrightarrow \frac{F_{\pi} g_{\eta^{\prime} N N}}{2 M_{N}} \cos \theta_{3} \frac{3}{\sqrt{6}} \longrightarrow \frac{\sqrt{3}}{2 M_{N}} \cdot \frac{F_{\pi}}{\sqrt{2}} g_{\eta^{\prime} N N}=\frac{\sqrt{N_{f}}}{2 M_{N}} f_{\eta^{\prime}} g_{\eta^{\prime} N N}
\end{aligned}
$$

Second term of Eq. (13) :

$$
\begin{aligned}
& \frac{F_{\pi} g_{\eta N N}}{2 M_{N} m_{\eta}^{2}}\left[-\sin \theta_{3} \frac{2 m_{K}^{2}+m_{\pi}^{2}}{\sqrt{6}}-\frac{2}{\sqrt{3}}\left(m_{K}^{2}-m_{\pi}^{2}\right) \cos \theta_{3}+\left(m_{K^{+}}^{2}-m_{K^{0}}^{2}-m_{\pi^{+}}^{2}+m_{\pi^{0}}^{2}\right)\right. \\
& \left.\times\left(\theta_{1} \cos \theta_{3}+\theta_{2} \sin \theta_{3}\right)\right]-F_{s} \widetilde{m}^{2} \frac{g_{\eta N N}}{2 m_{\eta}^{2} M_{N}} \sin \theta_{3} \\
& \stackrel{f_{\eta^{\prime}}=\frac{F_{\pi}}{\sqrt{2}}}{\longrightarrow} \frac{f_{\eta^{\prime}} g_{\eta N N}}{2 M_{N} m_{\eta}^{2}}\left[-\frac{2 m_{K}^{2}+m_{\pi}^{2}}{\sqrt{3}} \sin \theta_{3}-\frac{2 \sqrt{2}}{\sqrt{3}}\left(m_{K}^{2}-m_{\pi}^{2}\right) \cos \theta_{3}\right]-\frac{f_{\eta^{\prime}} g_{\eta N N}}{2 M_{N} m_{\eta}^{2}} \sin \theta_{3} \sqrt{3} \widetilde{m}^{2} \\
& \sin \theta_{3}=-\frac{2 \sqrt{2}\left(m_{K}^{2}-m_{\pi}^{2}\right)}{3 m^{2}}{\stackrel{\eta^{\prime}}{\sqrt{3}}}^{2}\left[\sin \theta_{3}\left(-\sqrt{3} \widetilde{m}^{2}-\frac{2 m_{K}^{2}+m_{\pi}^{2}}{\sqrt{3}}\right)-m_{\eta^{\prime}}^{2} \sin \theta_{3} \cos \theta_{3}\right] \frac{f_{\eta^{\prime}} g_{\eta N N}}{2 M_{N} m_{\eta}^{2}} \\
& \stackrel{f_{\eta}=f_{\eta^{\prime}}}{\longrightarrow} \frac{f_{\eta} g_{\eta N N}}{2 M_{N} m_{\eta}^{2}}\left[\sin \theta_{3}(1-\sqrt{3}) m_{\eta^{\prime}}^{2}+\sin \theta_{3}\left(\sqrt{3}\left(2 m_{K}^{2}-m_{\eta}^{2}\right)-\frac{2 m_{K}^{2}+m_{\pi}^{2}}{\sqrt{3}}\right)\right] \\
& f_{\eta} g_{\eta N N}=2 M_{N} g_{A}^{8} / \sqrt{6} \frac{g_{A}^{8}}{\sqrt{6} m_{\eta}^{2}}\left(\sin \theta_{3}(1-\sqrt{3}) m_{\eta^{\prime}}^{2}\right) \\
& \longrightarrow \frac{g_{A}^{8}}{\sqrt{6} m_{\eta}^{2}} \theta_{3}(1-\sqrt{3}) \sqrt{6} m_{\eta}^{2} \quad \longrightarrow \quad-\frac{1}{\sqrt{2}} g_{A}^{8} \theta_{3}
\end{aligned}
$$


Third term of Eq. (13) :

$$
\begin{aligned}
& \pm \frac{1}{2 M_{N}}\left\{F_{s} \widetilde{m}^{2} \frac{g_{\pi^{0} N N}}{m_{\pi^{0}}^{2}} \theta_{2}+\frac{F_{\pi} g_{\pi^{0} N N}}{m_{\pi^{0}}^{2}}\left[\sqrt{\frac{3}{2}} \frac{2 m_{K}^{2}+m_{\pi}^{2}}{3} \theta_{2}+\frac{2}{\sqrt{3}}\left(m_{K}^{2}-m_{\pi}^{2}\right) \theta_{1}+\delta m^{2}\right]\right\} \\
& \longrightarrow \pm \sqrt{\frac{3}{2}} g_{A}^{8} \theta_{2} \quad \text { (See Section 3.) }
\end{aligned}
$$

